Postwachstum

\section{Globaler Süden, Kapitalismus und die Zukunft von Degrowth}

\author{
Große Fragen wie ,Postwachstum für den Globalen Süden?‘ \\ und ,Degrowth im Kapitalismus?‘ standen im Mittelpunkt der \\ fünften Degrowth-Konferenz. Neben den inhaltlichen Diskus- \\ sionen ist nun auch eine Debatte über die Zukunft der Degrowth- \\ Bewegung entbrannt. Von Steffen Lange
}

D ie fünfte internationale DegrowthKonferenz für soziale Gerechtigkeit und ökologische Nachhaltigkeit fand im August in Budapest statt. Degrowth in einem osteuropäischen Land, ist das eine gute Idee?

Machen wir das Thema noch etwas größer: Sollte man das Konzept Degrowth auch auf Länder des globalen Südens übertragen? Dann noch eine der ganz großen Fragen: Ist Degrowth überhaupt innerhalb eines kapitalistischen Wirtschaftssystems möglich? Und was bedeuten die Antworten auf all diese Fragen für zukünftige Strategien und Aktivitäten der Degrowth-Bewegung?

\section{Degrowth-Konferenz in Osteuropa}

Vor etwa eineinhalb Jahren fiel die Wahl für die fünfte Degrowth-Konferenz auf Ungarn. Kurz zuvor hatte die bis dahin mit Abstand größte Konferenz in Leipzig stattgefunden. Im Nachklang hieß es, „diese Konferenz war so gut organisiert und so groß, dass es schwierig wird für nachfolgende Konferenzen“.

Damit war es für das Organisationsteam in Budapest eine Herausforderung, den Erwartungen gerecht zu werden. Das Team hat sich daraufhin für eine kleinere Konferenz entschieden, die sich bewusst auf wissenschaftliche Formate konzentriert, um in Ungarn ernst genommen $\mathrm{zu}$ werden (in Leipzig waren neben der Wissenschaft insbesondere viele Bewegungsakteure präsent).
Die Angst, dass nachfolgende Konferenzen nach Leipzig im Schatten der vorherigen Konferenz untergehen würden, hat sich nicht bewahrheitet. Allein schon deshalb, weil die Konferenz in Budapest einfach nicht vergleichbar war. Während man in Deutschland auf wachstumskritischen Diskussionen und vorherigen Konferenzen (z. B. Jenseits des Wachstums 2011) aufbauen konnte, war die Konferenz in Ungarn der Versuch, das Konzept "Degrowth“ in die dortige Debatte zu tragen. Denn in Ungarn waren die Terme „Degrowth“ oder „PostGrowth" bis dahin fast gänzlich unbekannt. Vor diesem Hintergrund hat die Konferenz in Budapest Erstaunliches erreicht: Sie fand in einem zentralen Universitätsgebäude statt, sie hat es geschafft, Größen der Degrowth-Szene nach Ungarn zu holen, sie fand Resonanz in lokalen und sozialen Medien und sie war wunderbar eng mit lokalen Initiativen verknüpft.

Ich kann nicht bewerten, inwiefern es geschafft wurde, wachstumskritische Diskussionen in die ungarischen Debatten zu tragen - und vielleicht ist es auch noch zu früh, dies zu bewerten. Eine andere Frage ist jedoch, ob die DegrowthDiskussionen (die vorrangig aus Frankreich, Spanien, Italien, Großbritannien, Deutschland usw. stammen) zur politischen und wirtschaftlichen Situation in Ungarn passen. Diese Frage konnte auf der Konferenz nur ansatzweise diskutiert werden - vor allem, weil dann doch der Großteil der Teilnehmenden aus Westeuropa kam.

\section{Degrowth im globalen Süden?}

Was hingegen stärker diskutiert wurde, war die Frage, ob das Konzept Degrowth auf Länder des globalen Südens übertragbar ist. Ashish Kotari, ein indischer Intellektueller und Aktivist, hat diese Debatte geprägt. Aus seiner Sicht sollten Verteter/innen von DegrowthKonzepten nicht versuchen, diese Bitte auf Länder des globalen Südens zu übertragen.

Erstens aus einer postkolonialen Machtperspektive: Aufgrund der Kolonialgeschichte würden in vielen Ländern des globalen Südens Konzepte aus dem Norden nach wie vor oftmals starkes Gehör finden und somit lokale Konzepte verdrängen. Als Folge verlören diese $\mathrm{Zu}$ lauf und Unterstützung. Zweitens aus einer inhaltlichen Sicht: Jede Region und jedes Land habe individuelle Verhältnisse. Ein Konzept für zukünftige Wirtschafts- und/oder Gesellschaftsformen wie Degrowth ließe sich daher nicht ohne Weiteres auf andere Regionen übertragen (siehe auch Kotari 2016). Zusammengenommen also sehr starke Argumente gegen eine Übertragung des Degrowth-Konzeptes auf Länder des globalen Südens. Die Alternative bestünde darin, mit Bewegungen (und deren Konzepten) aus den Ländern des globalen Südens zu kooperieren, im Sinne vieler Alternativen zum neoliberalen Kapitalismus. (Dies wird ja auch bereits öfters getan, siehe beispielsweise Burkhart et al. 2016.)

\section{Degrowth im Kapitalismus?}

Es scheint sich - zumindest unter den Teilnehmenden der Degrowth-Konferenzen - die geteilte Meinung herauszubilden, dass Degrowth innerhalb der bestehenden kapitalistischen Wirtschaftsstrukturen nicht funktionieren kann. Dieser Punkt wurde bereits auf vergangenen Konferenzen wiederholt vorgebracht. Neu für mich war auf dieser Konferenz, dass selbst die Vertreter/innen, die eine weniger kapitalismuskritische Sichtweise vertreten, diese 
Auffassung teilen. So argumentierte Daniel O’Neill (2016), einer der prominentesten Vertreter der steady state economy, dass Null- oder negatives Wachstum in einem kapitalistischen Wirtschaftssystem zu desaströsen Auswirkungen führe. Der Hauptgrund ist, dass positive Profitraten ohne gesamtwirtschaftliches Wachstum eine zunehmende Ungleichheit implizieren. Um allen Menschen in einer nicht wachsenden Wirtschaft ein gutes Leben zu ermöglichen, müssten also mindestens einige zentrale Institutionen des Kapitalismus - namentlich Profitgenerierung und Kapitalakkumulation - verändert werden.

\section{Strategien für Degrowth in der Zukunft}

Die Debatte nach zukünftigen Strategien ist nach der Konferenz stark aufgekommen. Mit einem Aufschlag haben Lange et al. (2016) argumentiert, dass neben den Konferenzen insbesondere (1) aktivistische Elemente, (2) der Aufbau von langfristig angelegten Institutionen in der Wissenschaft und anderswo und (3) Praxisprojekte weiter vorangetrieben werden sollten. Santarius (2016) und Beling (2016) betonen ebenfalls die Bedeutung des Aktivismus für die Zukunft der Degrowth-Bewegung.

Das meist genannte Beispiel ist hier die Zusammenarbeit zwischen Klimaaktivist/innen und Degrowth-Szene bei den Demonstrationen gegen die weitere Braunkohleförderung. Unter dem Titel ,Ende Gelände‘ ist es hier gelungen, das komplexe Konzept Degrowth auf konkrete Forderungen herunterzubrechen.

\section{Ausblick}

In Kürze wird bekannt gegeben werden, in welchem Land die nächste Degrowth-Konferenz stattfinden wird. Es bleibt abzusehen, ob und wie die Degrowth-Szene es schafft, neben den zweijährlichen Konferenzen und den Braunkohleprotesten noch weitere Leuchttürme der Bewegung zu etablieren.

\section{Literatur}

Beling, A. (2016): Some more Reflections on the Budapest Degrowth-Conference. Im Internet unter: https://www.degrowth.de/de/2016/10/ some-more-reflections-on-the-budapest-degrowth-conference/\#more-225348

Burkhart, C./Schmelzer, M./Treu, N. (2016): Einleitung - Degrowth in Bewegung(en) sucht nach Alternativen und Bündnissen. Im Internet unter: https://www.degrowth.de/de/dib/ degrowth-in-bewegungen/einleitung/

Kotari, A. (2016): Radical Ecological Democracy: Some More Reflections from the South on Degrowth. Im Internet unter: https://www.degrowth.de/de/2016/12/radicalecological-democracy-some-more-reflectionsfrom-the-south-on-degrowth/

Lange et al. (2016): Was wir von der 5. Internationalen Degrowth-Konferenz in Budapest mitnehmen. Im Internet unter: https://www. degrowth.de/de/2016/09/was-wir-von-der5-internationalen-degrowth-konferenz-inbudapest-mitnehmen/

O'Neill, D. (2016): Capitalism and (de)growth. Vortrag. Im Internet unter: https://youtu. be/7NTb_orrlP4?t=19m59s

Santarius, T. (2016): Die Degrowth-Bewegung ist bunt, kreativ und intelligent - aber noch wenig aktivistisch. Im Internet unter: http://www.postwachstum.de/die-degrowthbewegung-ist-bunt-kreativ-und-intelligentaber-noch-wenig-aktivistisch-20160906

\section{AUTOR + KONTAKT}

Steffen Lange ist wissenschaftlicher Mitarbeiter zu den Themen sozial-ökologische Wirtschaftspolitik und Postwachstumsökonomie am Institut für ökologische Wirtschaftsforschung (IÖW).

Institut für ökologische Wirtschaftsforschung, Potsdamer Str. 105, 10785 Berlin. Tel.: +49 3088459427 , E-Mail: steffen.lange@ioew.de

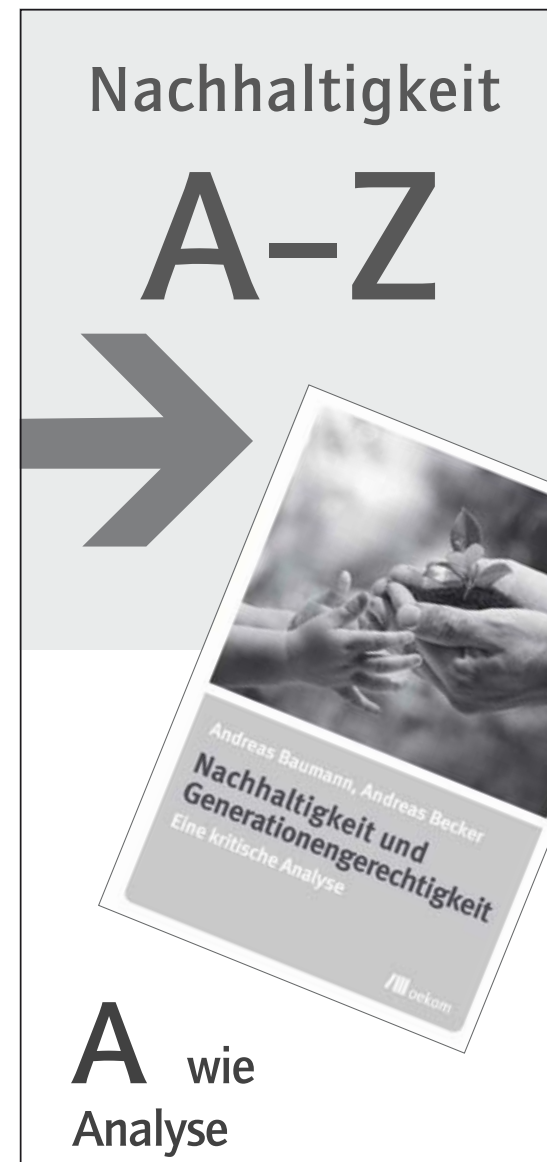

Die Begriffe "Nachhaltigkeit" und "Generationengerechtigkeit" sind als Schlagworte in aller Munde. Was genau verbirgt sich hinter den Konzepten der Nachhaltigkeit und der Generationengerechtigkeit? Wie unterscheiden sie sich voneinander? Worin liegen die jeweiligen Vor- und Nachteile? Und handelt unsere Gesellschaft heute nachhaltig und generationengerecht? Andreas Baumann und Andreas Becker bieten eine systematische Grundlage für nachhaltiges und generationengerechtes Handeln.

A. Baumann, A. Becker

Nachhaltigkeit und

Generationengerechtigkeit

Eine kritische Analyse

142 Seiten, broschiert, 16,95 Euro,

ISBN 978-3-86581-829-4

Erhältlich im Buchhandel oder versandkostenfrei innerhalb Deutschlands bestellbar unter www.oekom.de. Auch als E-Book erhältlich.

\section{/II oekom}

Die guten Seiten der Zukunft 